\title{
Littérature, histoire et religion dans l'empire moghol : à propos de trois ouvrages récents
}

Denis Matringe

\section{(2) OpenEdition \\ 12 Journals}

Édition électronique

URL : http://journals.openedition.org/assr/27229

DOI : $10.4000 /$ assr.27229

ISSN : $1777-5825$

Éditeur

Éditions de l'EHESS

\section{Édition imprimée}

Date de publication : 1 octobre 2015

Pagination : $129-146$

ISBN : 978-2-7132-2515-4

ISSN : 0335-5985

\section{Référence électronique}

Denis Matringe, «Littérature, histoire et religion dans l'empire moghol : à propos de trois ouvrages récents ", Archives de sciences sociales des religions [En ligne], 172 | octobre-décembre, mis en ligne le 26 avril 2018, consulté le 20 avril 2019. URL : http://journals.openedition.org/assr/27229 ; DOI : 10.4000 /assr.27229 


\title{
Denis Matringe
}

\section{Littérature, histoire et religion dans l'empire moghol : à propos de trois ouvrages récents}

\author{
À propos de : \\ ABU'L-FAZL, The History of Akbar, vol.1, éd. et trad. Wheeler \\ M. Thackston, Cambridge (Ma.), Murty Classical Library of India \\ et Harvard University Press, 2015, XXII +614 p. (cartes, notes, \\ glossaire, bibliographie, index). \\ BullHe SHAH, Sufi Lyrics, éd. et trad. Christopher Shackle, Cambridge \\ (Ma.), Murty Classical Library of India et Harvard University \\ Press, 2015, XXXVI + 443 p. (notes, glossaire, bibliographie, index). \\ SuRDAS, Sur's Ocean: Poems from the Early Tradition, éd. Kenneth \\ E. Bryant, trad. John Stratton Hawley, Cambridge (Ma.), Murty \\ Classical Library of India et Harvard University Press, 2015, \\ XLVIII + 1010 p. (notes, glossaire, bibliographie, index).
}

Quand les questions de chercheurs en sciences sociales du religieux travaillant sur l'aire culturelle européenne conduisent ceux-ci à utiliser comme sources des œuvres du passé considérées comme des classiques, qu'il s'agisse par exemple des commentaires d'Origène, des chansons spirituelles de Marguerite de Navarre ou des écrits de Fénelon, ils ont tout de suite à portée de main, à travers des collections comme les «Sources chrétiennes » (Le Cerf, Paris), les "Textes littéraires français » (Droz et Minard, Genève et Paris) ou «La Pléiade » (Gallimard, Paris), des textes rigoureusement édités, introduits et annotés, le cas échéant avec une traduction en vis-à-vis. La situation des spécialistes de l'Inde est bien différente. Alors que ce pays est riche d'immenses corpus littéraires en de nombreuses langues, le travail d'édition critique et de traduction entrepris dès l'époque de la colonisation britannique reste immensément lacunaire et parcellaire, et pour nombre de textes, périmé. Il convient donc de saluer comme une entreprise majeure le lancement de la Murty Classical Library of India. Cette dernière se propose en effet de publier, en graphie originelle et avec leur traduction anglaise en regard, des éditions scientifiques de grands textes d'une tradition multilingue s'étalant sur plus de trois mille ans, depuis les plus anciens hymnes védiques - composés au plus tard vers 1500 avant l'ère chrétienne, selon le 
consensus savant actuel - jusqu'à 1800 de l'ère chrétienne, quand commence à se faire sentir l'impact de la colonisation britannique.

Les cinq premiers volumes sont parus en cette année 2015 - des livres d'une grande élégance, imprimés sur un très beau papier, munis d'un marque-page, avec une couverture et une jaquette rouge framboise satiné à liseré doré, sur lesquelles le titre, le nom de l'auteur et celui de la collection ainsi que son logo, un éléphant stylisé vu de profil, figurent en deux nuances de doré, et celui de l'éditeur et du traducteur en blanc argenté. C'est à trois de ces ouvrages que la présente note est consacrée : l'Akbarnāma d'Abu'l-Fazl (chronique en persan de l'empereur moghol Akbar, $\mathrm{XVI}^{\mathrm{e}}$ siècle), les poèmes krishnaïtes en braj de Surdas (XVI ${ }^{\mathrm{e}}$ siècle) et les poèmes soufis en panjabi de Bullhe Shah (XVIII ${ }^{\mathrm{e}}$ siècle) ${ }^{1}$. On dira quelques mots de la nouvelle série dans laquelle ces volumes ont pris place avant de s'intéresser à leur contenu respectif, puis à leur utilité pour le travail en sciences sociales du religieux.

La mise en chantier de la Murty Classical Library of India (MCLI) et son développement futur ont été rendus possibles grâce à un don de 5,2 millions de dollars fait par Rohan Narayana Murty (né en 1983), citoyen indien, fils du richissime fondateur d'InfoSys Narayana Murthy (né en 1946) et docteur en informatique de l'université de Harvard qui gère la série ${ }^{2}$. Cette dernière est dirigée par un éminent indianiste étatsunien, Sheldon Pollock, titulaire de la chaire "Raghunathan » de sanskrit et d'études sud-asiatiques à l'université Columbia $^{3}$. Au sein du conseil éditorial qui sélectionne les textes à éditer et traduire ainsi que les chercheurs auxquels ils sont confiés, Sheldon Pollock, luimême en charge des littératures en langues indo-aryennes anciennes (sanskrit, pali, prakrits, apabhramsha, etc.), s'est entouré de trois des meilleurs spécialistes contemporains des littératures indiennes. Monika Horstmann, professeure émérite d'études indiennes modernes à l'université de Heidelberg, est en charge des littératures en langues néo-indo-aryennes (hindi, bengali, panjabi, marathi, etc.), qui se sont développées après l'an 1000. Sunil Sharma, professeur associé de littérature persane et comparée à l'université de Boston, s’occupe du persan, dont les premiers textes produits en Inde apparaissent au cours des premiers siècles du deuxième millénaire, et de l'ourdou, attesté depuis le $\mathrm{XV}^{\mathrm{e}}$ siècle. Enfin, David

1. Les deux autres volumes parus sont Anonyme, 2015 et Peddana, 2015.

2. Mukherjee, 2015, p. 41.

3. Sheldon Pollock a notamment écrit le fondamental The Language of the Gods in the World of Men: Sanskrit, Culture, and Power in Premodern India (voir bibl.), dirigé Literary Cultures in History: Reconstructions from South Asia (voir bibl.) et publié quatre volumes de traduction de poésie sanskrite. Il était en outre le codirecteur de la Clay Sanskrit Library (http: //www.claysanskritlibrary.org/), à laquelle il a été mis fin, et il est le fondateur et le directeur des séries "South Asia Across the Disciplines " (Universités de Californie, de Chicago et de Columbia, http://www.press.uchicago.edu/books/saad/index.html) et « Historical Sourcebooks in Classical Indian Thought » (Columbia, http://cup.columbia.edu/series/historical-sourcebooksin-classical-indian-thought). 
Shulman, professeur d'études humanistes et titulaire de la chaire "Renee Lang » à l'université hébraïque de Jérusalem, a la responsabilité des langues dravidiennes (tamoul, telugu, malayalam, etc.), dont les premiers textes remontent au milieu du premier millénaire. Certaines des éditions et traductions parues et à venir sont ou seront des premières : tel est ici le cas pour les poèmes de Bullhe Shah et de Surdas, édités scientifiquement et traduits intégralement en anglais pour la première fois.

Avec de vastes corpus dans plusieurs langues de ces diverses familles linguistiques, l'Inde présente la tradition littéraire plurilingue la plus complexe et la plus continue au monde. Mais comme le souligne Sheldon Pollock dans sa présentation de la MCLI, cette continuité a subi le double impact de la modernité coloniale puis de la globalisation contemporaine, avec un danger croissant pour l'Inde d'être de plus en coupée de son passé littéraire. Le hindi, par exemple, est aujourd'hui très éloigné de ses formes classiques dont l'une est ici représentée par les poèmes de Surdas. Le persan - langue d'Abu'l Fazl, dans laquelle l'Inde a produit plus que l'Iran du XV $\mathrm{VV}^{\mathrm{e}}$ au XVIII ${ }^{\mathrm{e}}$ siècle - a décliné dans sa patrie d'adoption à partir de la chute de l'Empire moghol et en a disparu au cours de la première moitié du XXe siècle, et le panjabi de Bullhe Shah, avec la partition du Panjab en 1947, a connu des fortunes bien différentes en Inde, où il est langue nationale de l'État du Panjab dans une forme standardisée, et au Pakistan, où il ne jouit d'aucun statut officiel ${ }^{4}$.

Mais que sont les "classiques" qui seront retenus pour figurer dans la MCLI ? Sheldon Pollock adopte à cet égard une position originale. Il commence par critiquer la conception d'auteurs comme Sainte-Beuve ou Eliot pour qui « classique » signifie à la fois universel et perpétuellement contemporain en soulignant qu'elle est en réalité occidentale, et donc " pas si universelle ni contemporaine après tout » :

«Qu'est-ce qui, selon nous, poursuit l'éditeur-en-chef de la série, rend des œuvres indiennes classiques? Ce pourrait être en fait leur résistance même à la contemporanéité et à l'universalité, c'est-à-dire leur capacité à communiquer la vaste variété du passé humain. Certes en bien des occasions ces livres nous apprendront quelque chose de notre commune humanité, mais ils nous offrent aussi un accès à des formes radicalement différentes de conscience humaine, élargissant par-là l'éventail des possibilités de ce qu'être humain veut dire ${ }^{5}$.

Il ne fait aucun doute que les trois livres dont il va être question maintenant, et qui proviennent de la période de l'Empire moghol (1526-1858), répondent bien à cette définition. Celui d'Abu'l-Fazl (1651-1602), chronique en persan consacrée à l'empereur Akbar (1542-1605, r. 1556-1605), présente la vision du pouvoir politique à l'époque moghole $^{6}$ : rappelons que le persan s'était imposé

\footnotetext{
4. Voir Pollock, 2015.

5. Pollock, 2015.

6. Pour une mise au point récente sur Akbar, voir Streusand, 2007-... (a).
} 
depuis le sultanat de Delhi (1206-1555) comme lingua franca et langue du gouvernement, de l'administration et de l'islam officiel en Inde du Nord. Quant aux poèmes de dévotion à Krishna composés en braj (vieux hindi littéraire occidental) par le saint poète hindou Surdas (seconde moitié du XvI siècle) et à ceux du mystique musulman Bullhe Shah (c. 1680-1750), écrits dans le panjabi dialectalement mixte typique des poètes soufis du Panjab à son époque, ils témoignent de formes de relation au divin inconnues en occident.

La tâche de présenter, de traduire et d'annoter l'Histoire d'Akbar (Akbarnāma) a été confiée à Wheeler M. Thackston, professeur émérite de persan et d'autres langues proches-orientales à l'université de Harvard, auteur de nombreux ouvrages destinés à l'étude du persan, de l'arabe, du turc et d'autres langues, et déjà traducteur des autobiographies du premier empereur moghol, Babur (14831530, r. 1526-1530), et du troisième, Jahangir (1569-1627, r. 1605-1627), respectivement grand-père et fils d'Akbar, ainsi que de trois Mémoires de Humāyun (1508-1556, r. 1530-1540 et 1555-1556), père d'Akbar ${ }^{7}$.

Abu'l Fazl est l'un des plus grands intellectuels indo-musulmans du XVI siècle, avec une vaste érudition dans les domaines du commentaire coranique, de la jurisprudence, de la philosophie et de la mystique islamiques ainsi que dans ceux de l'historiographie et des belles-lettres ${ }^{8}$. Il entre au service d'Akbar en 1575 et très vite joue un rôle de plus en plus important dans le programme politique de l'empereur. Les deux hommes méprisent les oulémas et se montrent particulièrement curieux des autres religions. Abu'l Fazl participe activement aux débats organisés par Akbar entre des représentants des sunnites, des chiites, des soufis, des hindous, des jaïns, des zoroastriens et des catholiques (des jésuites présents dans son empire). Il est sans doute le rédacteur du décret par lequel Akbar se proclame "souverain de l'islam » et "souverain juste » et s'arroge le droit de légiférer en matière religieuse. Abu'l Fazl est aussi un membre, et probablement l'inspirateur, de l'ordre religieux personnel d'Akbar appelé Dìn-i ilāhī (religion divine). Ce groupe d'élite consiste en individus reconnaissant Akbar comme guide spirituel supérieur à toute autre autorité religieuse. Enfin, Abu'l Fazl exerce d'importantes responsabilités politiques et militaires comme gouverneur d'une des provinces de l'empire et il anime le bureau impérial des traductions, supervisant la traduction en persan de nombreux classiques sanskrits, à commencer par la grande épopée du Mahābhārata.

Wheeler M. Thackston rappelle tous ces faits dans son introduction (p. VII-XXII), comme aussi qu'Abu'l Fazl périt assassiné par un chef de guerre hindou partisan de Salim, le fils rebelle d'Akbar qui finit par succéder à celui-ci après sa mort sous le nom de Jahangir. Mais il insiste tout particulièrement sur l'Akbarnāma.

7. Thackston, 1999,2002 et 2009.

8. Pour une remarquable mise au point récente à son sujet, voir Streusand, 2007-... (b). 
C'est en 1590 que la rédaction en fut confiée à Abu'l Fazl, à qui il était demandé, à travers l'histoire d'Akbar, d'écrire aussi celle des Timourides, l'empereur descendant de Tamerlan (1336-1405) par son père et de Gengis Khan (c. 1160-1227) par sa mère.

L'Akbarnāma s'est vite imposé dans tout le monde iranien et iranisé comme un modèle du genre historiographique en prose persane, avec ici et là quelques vers pour souligner des moments forts. Il est divisé en trois volumes (daftar) : le premier traite de la naissance et de l'ascendance d'Akbar, le deuxième du règne de l'empereur jusqu'en 1598 et le troisième, ou A' '̄n-i Akbarī (Règle d'Akbar), consiste en une encyclopédie de l'empire, avec une description détaillée de l'armée, de la cour, des provinces, de arts et métiers et de la pensée religieuse des hindous. L'ensemble manifeste un immense travail de recherche : comme le souligne Wheeler M. Thackston, Abu'l Fazl a eu accès aux travaux d'historiographes antérieurs, s'est entretenu avec de vieux serviteurs de Babur et Humayun et a travaillé sur des textes hindous, avec l'aide de traducteurs.

Pour sa traduction, Wheeler M. Thackston a utilisé l'édition de Calcutta de l'Akbarnāma, elle-même fondée sur dix manuscrits ${ }^{9}$. Abu'l-Fazl commence son ouvrage par une longue introduction dans laquelle il loue Dieu, la raison et la sagesse et explique la genèse de son travail (p. 2-37). Il en vient ensuite à la naissance d'Akbar (p. 39-79), à son horoscope selon les traditions grecque et indienne (p. 80-105), à la synthèse opérée par l'astrologue Azudduddaula Amir Fathullah Shirazi (p. 106-119) et aux questions d'interprétation (p. 120-145). Après avoir évoqué les premiers jours d'Akbar au milieu de ses nourrices, avoir donné le chronogramme de sa naissance et s'être une nouvelle fois félicité d'être le contemporain d'un être aussi exceptionnel, Abu'l-Fazl opère, pour évoquer la généalogie d'Akbar, un grand retour dans le passé, commençant par Adam et poursuivant à travers toute une galerie de personnages dont Noé, son fils Japheth, Türk, fils aîné de Japheth, et Tamerlan (p. 176-269). L'histoire du grand-père d'Akbar, Babur, et de son père, Humayun, sont ensuite traitées en détail (respectivement p. 282-385 et p. 386-571). Les dernières pages de ce premier volume de l'Akbarnāma sont consacrées à la réception par Humayun de la nouvelle de la naissance de son fils (p. 558-565) et aux deux premiers miracles accomplis par Akbar (p. 566-571). Alors que le prince est dans son huitième mois, sa principale nourrice, Jiji Anäkä, est accusée de sorcellerie par ses rivales jalouses. Le bébé se met alors à parler et dit à Jiji de ne pas s'inquiéter, car il sera toujours à ses côtés. Quelques jours plus tard, ledit bébé se trouve au sein du cortège qui accompagne l'empereur Humayun pour une partie de chasse. "Soudain, écrit Abu'l-Fazl, apparut sur le chemin un terrible serpent, qui aurait causé de l'effroi au plus courageux. Sa Majesté accomplit alors le miracle de Moïse, et sans

9. Abū 1-Faẓl-i'Allāmī, 1873-1887. 
prendre le temps de réfléchir, alla droit au serpent, l'attrapa par la queue et, de sa main bénie, le jeta au loin ${ }^{10}$. "

Dans le chapitre de son introduction intitulé "Language and style of the Akbarnama » (p. 13-16), Wheeler M. Thackston souligne deux points très importants pour la lecture du livre d'Abu'l-Fazl. D'une part, la mystique islamique imprègne de bout en bout non seulement le vocabulaire et le style de l'Akbarnāma, mais aussi toute sa philosophie, Akbar finissant par apparaître comme l' "Homme parfait » de la mystique islamique, image de Dieu sur terre. D'autre part, Abu'lFazl va encore plus loin et décrit Akbar comme la manifestation de la lumière divine apparue dans le sein d'Alanqoa, ancêtre mythique de Gengis Khan et de Tamerlan (p. 42-43). Wheeler M. Thackston rappelle aussi que l'Akbarnāma est un texte de parti pris, dans lequel tous ceux qui s'opposent à l'empereur ou se rebellent contre lui sont condamnés en termes très durs.

La traduction de Wheeler M. Thackston est remarquable. Tout en étant éminemment fluide, elle rend compte du caractère très orné de la prose d'Abu'lFazl et restitue admirablement le caractère grandiose de l'ouvrage. Voici à titre d'exemple la traduction du passage dans lequel Abu'l-Fazl en vient à évoquer le début de son entreprise (p. 162):

When this luminary of fortune rose, the writer of this amazing book was still laboring in the recesses of nonexistence under the burden of regret for not yet existing and worshipping God. How can he express his gratitude for the most magnificent gift of living during the time of this truly and metaphorically great man and for being one of those gazed upon with the eye of patronage and favor? A hundred thanks that, before even the royal horoscope was taken into consideration and the marvels of its glories were realized, he attained perfect piety and sovereignty, which are higher than the bases of astrologers' cognition, and was nursed by divine power.

Dans l'A'’'n-i akbari, Abu'l-Fazl consacre trois chapitres respectivement aux savants, aux poètes et aux musiciens de l'époque d'Akbar. Si plusieurs des cent quarante savants et certains des trente-six musiciens mentionnés portent des noms hindous, il n'en va pas de même des poètes qui ont, eux, tous des noms musulmans. Un Surdas figure bien au nombre des musiciens, mais, comme le fait remarquer John Stratton Hawley (p. XII), il est hautement invraisemblable qu'il s'agisse de l'auteur des poèmes traduits par cet universitaire étatsunien, professeur de religion au Barnard College de l'université Columbia et éminent spécialiste des littératures hindoues de dévotion en hindi aux XV ${ }^{\mathrm{e}}-\mathrm{XVI}^{\mathrm{e}}$ siècles ${ }^{11}$. L'immense poète que fut Surdas, s'il avait été connu d'Abu'l-Fazl, aurait figuré parmi les poètes et son excellence eût été soulignée.

10. L'allusion à Moïse renvoie au passage du Livre de l'exode (IV.1-5) où Moïse, lors de la révélation de sa mission, s'entend intimer l'ordre de jeter son bâton sur le sol et le voit se transformer en serpent. Dieu lui ordonne alors d'attraper ce serpent par la queue, lequel redevient aussitôt un bâton (allusion à cet épisode dans le Coran en 26.32).

11. Hawley, 2015. Signalons aussi, parmi des études fondamentales de John Stratton Hawley sur la bhakti et Surdas, ses livres de 1981, 1983, 1984, 2004, 2005 et 2009. 
Surdas, au demeurant, ne fut pas, loin s'en faut, le seul très grand poète hindou contemporain d'Akbar. Il y en eut bien d'autres, à tel point qu'une pionnière française des études sur les poésies hindoues de dévotion en hindi, Charlotte Vaudeville (1918-2006), dans un livre consacré à Surdas, a pu parler de " renaissance hindoue " à l'époque d'Akbar ${ }^{12}$. Le rapprochement entre ce fait et son temps n'est pas fortuit. En effet, dans le cadre du programme politique d'Akbar si magistralement explicité par Abu'l-Fazl, l'empereur se voulait souverain de tous les Indiens de ses territoires, qu'ils fussent hindous ou musulmans. Pour Akbar, qui épousa une princesse hindoue et dont le principal général était un maharadja hindou (tous deux rajasthanais), la distinction entre hindous et musulmans n'importait pas. Abu'l-Fazl, au demeurant, s'attache à démontrer dans la longue dernière partie de l'A' 'in-i Akbarī que tout comme les musulmans, les hindous sont au fond monothéistes.

Ce ne sont pas de vains mots et Akbar fut un mécène autant pour les artistes hindous que pour les artistes musulmans, de même qu'il patronna la construction aussi bien de temples hindous que d'établissements soufis et de mosquées. Cette universelle bienveillance favorisa considérablement l'épanouissement d'une nouvelle forme de vie religieuse en Inde du Nord: le développement de sectes initiatiques (sampradāya), caractérisées par la révélation reçue d'un dieu par un fondateur, l'enseignement de guru à disciples et la pratique d'une forme de théisme appelée bhakti, dévotion aimante totale à une divinité suprême avec laquelle le dévot (bhakta) entretient une relation personnelle. John Stratton Hawley, l'un des savants qui a le plus contribué à l'étude de la bhakti en général et de Surdas en particulier, a consacré de longs développements à ce lien entre pouvoir moghol et épanouissement de la bhakti dans son dernier livre intitulé A Storm of Songs: India and the Idea of the Bhakti Movement ${ }^{13}$. Tout le quatrième chapitre de l'ouvrage est précisément consacré, dans cette perspective, au pays braj, région située autour de l'ancienne cité de Mathura, à quelque cent cinquante kilomètres au sud-est de Delhi, traversée par la Yamuna et où est parlé un dialecte occidental du hindi lui aussi appelé braj.

Le pays braj est vénéré en Inde comme étant la contrée où naquit et grandit Krishna, avatāra de Vishnu, folâtrant à Vrindavan parmi les vachères (gopĩ) et accomplissant exploits et miracles ${ }^{14}$. Dès le début du XVI ${ }^{\mathrm{e}}$ siècle, le Braj commença à attirer de nombreux dévots de Krishna. Ces derniers en effet voyaient dans le Braj non seulement le pays des enfances et de certains des hauts faits de Krishna - un pays dont chaque bosquet, chaque tertre et chaque étang était lié à un épisode de la geste de leur Dieu (de tout ce que l'on appelle son «jeu » [Krș̣na-līlā] -, mais aussi, et pour cette raison même, une parcelle de la Terre où

12. Soûr-Dâs, 1971, p. 11.

13. Cambridge, Harvard University Press, 2015.

14. Sur le braj comme pays de Krishna, voir notamment Vaudeville, 1976 et Entwistle, 1987. 
il était possible d'être plus intimement en contact avec lui. D'importantes sectes vishnuïtes s'implantèrent ainsi dans le Braj, au nombre desquels le puștimārga (voie de la grâce), comme s'appelle le sampradāya qui se rattache à l'enseignement de Vallabha (1479-1531) - généralement appelé Vallabhacharya (Maître Vallabha) -, brahmane Telugu établi à Varanasi (Bénarès) dont les petits-fils contribuèrent à répandre l'ordre initiatique en Inde du Nord. La liturgie du puștimārga consiste essentiellement dans le chant de poèmes de dévotion composés par huit poètes, les huit "sceaux »(aștachāpa), au premier rang desquels Surdas, qui fleurit au XVI $\mathrm{e}^{\mathrm{e}}$ siècle mais dont les dates de vie sont inconnues (tradit. 1479-1584 !).

Le volume de la MCLI consacré à ce poète comporte deux introductions : celle du traducteur suivie de celle de l'éditeur du texte. C'est le premier, John Stratton Hawley, qui présente Surdas, connu seulement par ses poèmes et par les hagiographies, à commencer par celle écrite par l'un des petits-fils de Vallabhacharya, Gokulnath, au sein d'un recueil intitulé Caurasī Vaiṣnava kī vārtā, "Présentation des quatre-vingt-quatre Vaishnavas» (dévots de Vishnu). Surdas aurait été aveugle, mais on ne sait trop s'il l'était de naissance ou s'il le serait devenu, lors de son initiation par Vallabhacharya, à tout ce qui n'était pas Krishna : le maître aurait transféré en lui à cet instant tout le contenu et le sens $\mathrm{du}$ grand texte sanskrit du $\mathrm{X}^{\mathrm{e}}$ siècle qui a établi la version canonique de la geste krishnaïte, le Bhāgavata-purāna.

S'il existe un corpus vallabhite de Surdas, centré sur la vie de Krishna dans le Braj, celui-ci n'est pas le seul, et plutôt que parler de Surdas, il est préférable, rappelle John Stratton Hawley, de faire référence à une tradition de Surdas, le nombre de poèmes figurant dans les manuscrits n'ayant cessé de croître au fil des siècles, au fur et à mesure que se répandait en Inde du Nord la bhakti krishnaïte. On passe ainsi de 239 poèmes attribués à Surdas dans le manuscrit le plus ancien, daté de 1582, à quelque 10000 dans un manuscrit du XIX siècle - l'attribution résultant de l'insertion par le poète de son nom, en guise de "sceau " (chāpa), dans le dernier vers de chaque poème. Si nombreux sont les poèmes attribués à Surdas que dès le XVII ${ }^{\mathrm{e}}$ siècle, les manuscrits s'intitulent Sūrasāgara, "Océan (des poèmes) de Sur(das) ». À propos de ce mouvement, John Statton Hawley estime qu' «il vaudrait mieux penser le Sūrasāgara non comme un océan mais comme une rivière - gagnant en force au fil du temps, mais devenant aussi plus lente et perdant une bonne partie de la pureté qu'il était possible de goûter plus haut en amont » $(\mathrm{p} . \mathrm{XV})$. La dernière partie de cette première introduction est consacrée à un résumé de la geste krishnaïte et à l'évocation des épisodes les plus présents dans le corpus ancien de Surdas, comme ces « chants de l'abeille » (bhramargìta) Udho, messagère de Krishna auprès des vachères qui souffrent d'être séparées de leur aimé une fois qu'il a quitté Vrindavan pour Mathura. Krishna leur demande de pratiquer un yoga leur permettant de prendre conscience de sa divine présence avec elles et en elles - mais elles ne veulent rien entendre : 
It is a dirty trick, this yoga,

and it won't sell in Braj.

Who is going to let her pearls

be auctioned off for radishes leaves?

Udho, that's the measure of success

your tradesmanship will meet $[\ldots]^{15}$.

Kenneth Bryant prend ensuite le relais pour exposer d'une part la manière dont il a établi son édition du texte, et d'autre part pour proposer au lecteur une analyse très pointue de la métrique des poèmes de Surdas et du rapport de cette dernière avec le rythme musical (tāla) sur lequel ils sont destinés à être chantés. Comme tant d'autres poèmes de l'univers de la bhakti, les poèmes de Surdas ont d'abord circulé oralement - et il en va encore de même aujourd'hui -, si bien que les "manuscrits » sont en fait, avant tout, des notations de chants devenues au fil des siècles des recueils de plus en plus importants. La conséquence éditoriale tirée de ce fait par Kenneth Bryant est qu'il est impossible d'imaginer atteindre un Urtext des poèmes de Surdas ou de considérer, comme en philologie classique, qu'un manuscrit tardif pourrait être la copie du texte originel. Dans ces circonstances, écrit-il, " la seule indication qu'un poème soit ancien est qu'il soit présent dans un manuscrit ancien» (p. XXIX). En conséquence, son parti-pris éditorial a consisté à ne retenir que les poèmes présents dans les manuscrits datés de l'époque où vécut Surdas, soit un total de 433 (et, imagine-t-on, quand un texte apparaît dans plusieurs manuscrits, à retenir la version du manuscrit le plus ancien). Ces poèmes sont classés dans le livre selon la chronologie de la geste krishnaite, avec trois sections additionnelles concernant respectivement le mythe de l'autre avatāra majeur de Vishnu, Rama, les suppliques et les louanges du poète, et les rivières sacrées.

La seconde partie de cette deuxième introduction est très technique, mais elle permet à l'éditeur du texte d'une part d'insister sur le fait que fondamentalement, les poèmes de Surdas sont destinés au chant, et d'autre part de justifier sa disposition sur la page des vers en hémistiches comme étant plus apte à faire ressortir non seulement le rythme et la respiration du chant, mais aussi à mettre en valeur la répétition comme un refrain, à la fin de chaque strophe, des derniers mots de l'unique hémistiche en lequel consiste le premier vers de chaque poème, qui en est précisément le refrain.

Ce livre est le résultat de quarante années de travail (p. XXXVIII) ! Les chercheurs tout comme les personnes ayant à cœur l'humanitas planétaire et, pour reprendre les mots de Sheldon Pollock cités plus haut, « des formes radicalement différentes de conscience humaine ", ont désormais à leur disposition un corpus fiable et remarquablement traduit de la poésie de Surdas - auteur fondamental

15. Début du poème 278, p. 469. 
pour aborder la dévotion à Krishna si répandue et si vivante non seulement en Inde du Nord mais, désormais, dans le monde entier ${ }^{16}$.

Si le XVI ${ }^{\mathrm{e}}$ siècle dans lequel voisinent, sous le patronage d'Akbar et de sa noblesse religieusement plurielle, des auteurs de la stature d'Abu'l-Fazl et Surdas est une époque où l'Inde du Nord est politiquement intégrée comme jamais auparavant, la première moitié du XVIII ${ }^{\mathrm{e}}$ siècle, où Bullhe Shah vécut sa vie d'adulte, offre un tout autre tableau. L'Empire moghol, déjà ébranlé par guerres et rébellions à l'époque de son dernier grand souverain, Aurangzeb (1618-1707, r. 1658-1707), voit son autorité contestée et des États successeurs prendre forme à sa périphérie. Dès les années 1720, des pouvoirs locaux se sont consolidés dans le Deccan à Hyderabad ainsi que dans le nord au Bengale et dans la région de Laknau (Lucknow), tandis qu'une confédération marathe entreprend de vastes conquêtes et s'en prend même à Delhi. En 1739, le Persan Nadir Shah (1688-1747, shah d'Iran de 1736 à 1747) ravage le Panjab et met Delhi à feu et à sang. Moins de dix ans plus tard, c'est au tour d'Ahmad Shah Durrani (1722-1772, r. 17471772), premier souverain de l'Afghanistan moderne, de lancer des razzias meurtrières dans le Panjab, qui dès 1754 est totalement hors de contrôle pour le pouvoir moghol, comme le sont depuis quelques années déjà le Sind et le Gujarat.

Dans la tourmente du Panjab, de nouveaux acteurs jouent un rôle de plus en plus affirmé, les sikhs, qui en conquièrent ville après ville et finissent par y créer un royaume unifié avec Lahore pour capitale en 1799. Les sikhs, qui font remonter leur origine comme groupe religieux aux disciples d'un saint poète bhakta du Panjab nommé Nanak (1469-1539), ont connu une évolution saisissante, d'un paisible groupe de dévots au XVII siècle à une communauté animée par un ordre initiatique et militant formé en son sein en 1699 sous l'égide de leur dernier guru, Gobind (1666-1708).

Dans les villes et les villages de ce Panjab où l'autorité politico-religieuse impériale s'est délitée et où les affrontements armés sont incessants vivent ensemble hindous, sikhs et musulmans. Dans les campagnes, ascètes shivaites pratiquant le yoga, bhakta chantant leur kīrtan (chant collectif d'hymnes de dévotion) et poètes soufis composant des poèmes dans la langue locale sont des figures bien connues. Bullhe Shah est l'un de ces derniers, le plus grand et le plus fameux. Pour rendre justice à un tel auteur, il fallait tout l'immense savoir, toute la sensibilité poétique et tout le talent littéraire de Christopher Shackle, professeur émérite à la School of Oriental and African Studies, grand spécialiste des langues et des littératures de l'Inde du Nord, et le meilleur connaisseur des cultures littéraires du Panjab ${ }^{17}$.

16. Voir par exemple dans cette livraison d'Archives le compte rendu par Catherine Clémentin-Ojha de Richardson, 2014.

17. On doit à Christopher Shackle des outils et des études indispensables pour aborder les langues et les littératures du Panjab : mentionnons notamment deux grammaires, l'une du panjabi standard (1972) et l'autre de la langue parlée dans la région de Multan ou sirāiłki (1976), 
Christopher Shackle revient sur certains aspects du contexte évoqué ci-dessus dans l'introduction de son livre (p. VIII-XV), après avoir dit ce qu'il est possible de savoir de la vie du poète, qu'il s'agisse de sa famille appartenant au plus prestigieux groupe social des musulmans indiens - les Sayyid qui se prétendent descendants de Muhammad -, de son éducation, de sa formation en mystique islamique, notamment par son maître bien-aimé, le soufi de l'ordre qādirī Inayat Shah, issu la modeste caste des jardiniers $(m \bar{a} l \bar{\imath})$, et de ses dernières années, passées à Kasur, aujourd'hui dans le Panjab pakistanais (p. VII-VIII) ${ }^{18}$. Il relève au passage un fait important pour comprendre la popularité de la poésie de Bullhe Shah de nos jours : dans un monde indien où, comme le souligne aussi Sheldon Pollock, les nouveaux horizons d'attente culturels introduits par le colonialisme ont contribué à approfondir la rupture avec les littératures du passé, ce sont les classiques du XVIII ${ }^{\mathrm{e}}$ siècle, écrits dans une langue encore compréhensible, qui " peuvent aider à donner un sens à un présent culturellement fracturé » (p. IX). Tel est précisément le cas de la poésie de Bullhe Shah, écrite dans un panjabi "simple et direct ", et qui, malgré la partition du Panjab entre Inde et Pakistan et les drames dont elle s'est accompagnée, continue à évoquer pour les Panjabis des deux côtés de la frontière " une vision magique originaire d'une ancienne unité intemporelle, transcendant les divisions modernes qui troublent le présent» (ibid.).

Revenant ensuite sur les traits communs à la bhakti dans sa version sikhe et au soufisme, Christopher Shackle met en regard de la tendance de certains intellectuels à dénier la réalité des différences entre hindouisme et islam l'enracinement de la poésie de Bullhe Shah dans cette dernière religion, rappelant au passage les traits saillants de l'histoire du soufisme en Inde et celle de ses pratiques, rituelles (notamment le chant de poèmes) et linguistiques (rôle dominant du persan, recours au panjabi par les poètes soufis [p. XI-XIII]). Après la conquête du Panjab par les armées de la Compagnie britannique des Indes orientales au terme de deux guerres (1846 et 1849), le persan qui avait été la langue officielle du royaume sikh du Panjab (1799-1849) est rapidement remplacé comme langue d'administration et d'éducation supérieure et comme langue littéraire par l'ourdou et l'anglais. Mais dans le même temps, et alors que le réformisme musulman s'implante au Panjab et bientôt aussi le nationalisme politique musulman, des éditeurs s'attachent à publier les œuvres de Bullhe Shah et d'autres poètes soufis

deux articles de sociolinguistique consacrés respectivement à chacune de ces deux langues (1970 et $1977 \mathrm{a}$ ), une grammaire et un dictionnaire pour lire la littérature en sant-bhāṣā (1983 et 1995a), deux remarquables séries d'articles portant, pour l'une, sur certains aspects particuliers de la langue et des styles de l'Ādi Granth (1977b, 1978a, b et c), et pour l'autre sur divers aspects de la littérature musulmane en panjabi (1978d, 1992, 1993, 1995b, 2000, 2006, 2007 et 2011), plusieurs traductions $(1983,1985,2005)$ et des entrées en cours de parution sur les poètes soufis panjabis dans la troisième édition de l'Encyclopaedia of Islam.

18. La Qādiriyya est un ordre soufi très répandu dans le monde musulman. 'Abd al-Qadir Jilani (1077 ou 1078-1166), juriste et savant de Baghdad, en fut construit, après sa mort, comme le fondateur éponyme. 
panjabis du passé ou contemporains. Dans un tel contexte, Bullhe Shah se retrouve sujet « à toute une variété d'interprétations » reposant souvent sur " des lectures hautement sélectives de sa poésie» (p. XIV). Comme le note Christopher Shackle, son édition des œuvres complètes du poète avec leur traduction en anglais " peut aider à corriger certains biais qui ont trop souvent brouillé les manières de comprendre Bullhe Shah» (p. XV).

Après ces très utiles mises au point, Christopher Shackle procède à une présentation méthodique de la poésie de Bullhe Shah (p. XV-XXVIII). Concernant la forme, le genre principal du corpus est la $k \bar{a} f \bar{\imath}$, qui a beaucoup en commun avec le pada de la poésie religieuse de l'Inde du Nord - celle de Surdas notamment : un poème consistant en un refrain et des strophes rimées à métrique quantitative, dont la dernière comporte la « signature » du poète. La structure rimique privilégiée par Bullhe Shah suit un schéma A (refrain), BBBA, CCCA, etc. (strophes). On trouve aussi dans l'œuvre préservée de Bullhe Shah un poème des sept jours, un poème des douze mois, un poème didactique dit " des quarante nœuds ", en référence à la cordelette à nœuds utilisée pour compter les jours avant une occasion importante, et un autre dit "des trente lettres ", dont chaque strophe commence, en suivant l'ordre alphabétique, par une lettre de l'alphabet arabe. Viennent enfin de courtes pièces, consistant pour la plupart en deux vers $($ doha $\bar{a})-$ un type poétique très fréquent dans l'Inde du Nord de l'époque de la première modernité. En voici un exemple (p. 353), dans lequel se manifeste la sensibilité de Bullhe Shah au contexte de pluralité dans lequel il vivait et qui signifie que pour un maître parfaitement réalisé, les différences entre religions ne comptent plus :

Bullha, who is that supreme friend of yours? The Qur'an is in his hand, and yet he wears the sacred thread ${ }^{19}$.

Christopher Shackle en vient ensuite (p. XIX-XXVIII) à l'analyse thématique des poèmes de Bullhe Shah. Il rappelle que les vers de critique sociale sur lesquels ont insisté nombre d'intellectuels engagés de la période récente, pour vifs qu'ils soient, n'en restent pas moins très minoritaires au sein du corpus. L'inéluctabilité de la mort est par contre un motif récurrent, souvent symbolisé par le mariage et la nécessité pour la fiancée de bien préparer sa dot (qui représente les bonnes actions du fidèle) avant son départ pour la maison de son mari (qui symbolise Dieu). De fréquentes citations coraniques, rendues par des italiques dans la traduction, enracinent la poésie dans la vision islamique de l'homme, du cosmos et de Dieu. Dans ce cadre, Bullhe Shah se révèle un poète de l'unicité de l'Être (waḥdat al-wujūd, en arabe), doctrine qui remonte en islam à l'enseignement du grand mystique d'origine andalouse Ibn al-'Arabi (m. Damas, 1240). Il s'agit là

19. Le cordon rituel que reçoivent lors de leur initiation les membres mâles des trois classes supérieures de la société brahmanique : brahmanes (classe sacerdotale), kșatriya (rois et guerriers) et vaiśya (fermiers, marchands, prêteurs et artisans). 
pour lui, explique Christopher Shackle, d'un processus dynamique dans lequel l'amour - le pur amour - joue un rôle fondamental, et avant tout l'amour du poète pour son maître spirituel Shah Inayat, à travers lequel il accède à Dieu. La diversité des émotions religieuses liées à ce pur amour s'exprime parfois à travers l'évocation de grandes figures du soufisme comme Mansur Hallaj, martyrisé à Bagdad en 920, ou à travers de grands mythes hindous, au premier rang desquels celui de Krishna. Mais elle s'exprime surtout par l'utilisation symbolique d'histoires d'amour, arabes comme celle de Leila et Majnun ou, le plus souvent, typiques de la vallée de l'Indus, comme la célèbre légende panjabi des amours de Hir et de son amant Dhido Ranjha, contrariés par les implications du système des castes. Le poète identifie alors son âme à l'héroïne, le héros symbolisant Dieu. Voici par exemple, dans la traduction de Christopher Shackle, le refrain d'une $k \bar{a} f \bar{\imath}$ qui, tout en chantant la fusion de l'âme-amante et de son Aimé divin, fait référence au principal exercice spirituel des soufis, le $\underline{d} \underline{b} i k r$, répétition solitaire ou en groupe, à haute voix ou mentalement, d'un ou plusieurs nom(s) de Dieu ou d'une litanie, et qui peut impliquer musique et/ou dance ( $k \bar{a} f \bar{\imath} 145$, p. 249) :

Through repeating "Ranjha, Ranjha!", I have myself now become Ranjha. Call me Dhido Ranjha, let no one call me Hir.

Une note sur le texte et la traduction (p. XXXI-XXXVI) commence par faire l'histoire des éditions des poèmes de Bullhe Shah (dont il n'y eut, redisons-le, jamais d'édition complète), non sans évoquer les problèmes liés à l'absence de standardisation de l'orthographe du panjabi. Christopher Shackle explicite ensuite les partis-pris de son édition et de sa traduction. Son édition est fondée sur les meilleures éditions partielles existantes et les $k \bar{a} f \bar{\imath} \mathrm{s}$ y sont classées selon l'ordre alphabétique du premier mot du refrain dans l'écriture gurumukhī-retenue par l'éditeur-traducteur car beaucoup mieux adaptée à la notation du panjabi ${ }^{20}$. À propos de sa traduction, Christopher Shackle écrit ceci (p. XXXIII-XXXIV) :

Only a very fine verse translation might hope to convey the ecstatic tone of much of Bullhe Shah's poetry, although even the most gifted English poet could hardly hope to reproduce the many rhymes that are so defining a characteristic of the kāfī [...]. In keeping with the style of the Murty Library, the present translation of the full corpus therefore tries only to convey the sense of the poetry in a consistent style of plain English prose that aims to steer a middle path between off putting formality and jarring colloquialism.

Cette prose est en quelque sorte organisée en versets selon les strophes. Le traducteur sensible et talentueux qu'est Christopher Shackle est parvenu à lui insuffler de bout en bout l'aisance naturelle et la grâce aérienne qui caractérisent la poésie de Bullhe Shah tout autant que son ton extatique. Il a su à tout moment trouver le mot juste, le tour approprié, l'expression la mieux à même de véhiculer l'émotion se dégageant du texte original, et à travers cette traduction, le lecteur

20. La gurumukhī est l'écriture dans laquelle les sikhs écrivent le panjabi, noté en écriture arabe modifiée par les musulmans et dans l'écriture devanāgarì du hindi par certains hindous. 
qui ignore tout du panjabi recevra dans l'essentiel de son étincelante fraîcheur cette poésie du XVIII ${ }^{\mathrm{e}}$ siècle indien, les notes de fin et le glossaire lui permettant de ne rien manquer des allusions et des références du poète. Voici à titre d'exemple la traduction de la strophe consacrée au premier mois de la mousson (sāvan, p. 289) dans le poème des douze mois de Bullhe Shah. Comme le souligne en note le traducteur (p. 420), il est remarquable que le poète ait choisi de conclure son poème sur les deux mois de la mousson, qui ne sont pas les derniers du calendrier indien, mais ceux de l'événement tant attendu de l'arrivée des pluies, et qui, dans la poésie de l'Inde du Nord des XV $-\mathrm{XVIII}$ e siècles, sont chantés comme ceux de sentiments intenses - souvent en liaison avec le retour à la maison des maris après des mois de travail comme journaliers loin du village :

The clouds look lovely in Savan, the Creator looks lovely in the heart. "In every place Inayat dwells", calls the papīhā bird ${ }^{21}$.

The songs of the rainy season ${ }^{22}$ sound lovely throughout Savan. My enemies suffer pain and depart. The boys play and the girls sing. My house is filled with delights. My hopes are fulfilled.

The Lord has fulfilled my hopes. I have gazed upon him in love. My girlfriends have come to congratulate me. I say to Shah Inayat: "Lord, my hopes are fulfilled".

Après avoir tenté de contextualiser et de présenter le contenu de ces volumes de la MCLI consacrés respectivement à la principale chronique historiographique de l'empereur Akbar, à l'un des plus importants recueils de poésie krishnaïte de l'Inde du Nord au XVI ${ }^{\mathrm{e}}$ siècle et au corpus le plus représentatif de la poésie soufie en panjabi, je voudrais à présent chercher à mettre en lumière en quoi ces trois ensembles de textes peuvent intéresser les sciences sociales des religions en revenant de manière synthétique sur des questions de statut des langues, de jeux d'échelles ainsi que de dynamiques et de pratiques des groupes religieux, en liaison avec des formes de pouvoir et d'autorité.

Abu'l-Fazl, Surdas et Bullhe Shah sont trois auteurs de l'Inde moghole, c'està-dire d'une Inde dans laquelle le persan, importé de l'Asie centrale iranisée dès les premières conquêtes musulmanes au $\mathrm{X}^{\mathrm{e}}$ siècle, est la langue du pouvoir politique ainsi que celle des autorités religieuses que celui-ci patronne et qui le légitiment. Connaître cette langue est obligatoire pour qui veut obtenir un poste dans la machinerie impériale. Quant à accéder à l'entourage immédiat du souverain, cela suppose une maîtrise littéraire parfaite du persan : tel était le cas pour Abu'lFazl $^{23}$. Mais comme dans l'Inde d'aujourd'hui, chacune des régions de l'Empire moghol a sa propre langue, indienne cette fois, qui peut être le véhicule d'une ou de plusieurs traditions littéraires. Il existait une littérature krishnaïte en braj, représentée dans ce qui précède par les poèmes de Surdas, et aussi, au Panjab, des

21. The common hawk-cuckoo, Hierococcyx Varius (note du traducteur).

22. Literally "songs in Rag Malhar ", the musical mode associated with the rains (note du traducteur).

23. On retrouve là le phénomène si bien étudié par Cohn, 1996. 
littératures sikhe et islamique en panjabi, langue qui, dans nos textes, est celle de Bullhe Shah. Pour le braj de Surdas, c'est l'installation dans le Braj de puissantes sectes vishnuïtes, au premier rang desquelles le puștimārga, et le patronage qu'elles accordent à la poésie krishnaïte composée dans cette langue qui en favorisent la diffusion dans toute l'Inde du Nord, indépendamment même des courants religieux qui l'ont portée. C'est ainsi qu'à partir du XVIII siècle, le braj devient l'une des langues religieuses utilisées par les sikhs, à côté de la sant-bhāṣā, du panjabi et du persan ${ }^{24}$. Inversement, le panjabi des poèmes de Bullhe Shah, dont l'ordre soufi qādirī assure la diffusion dans le Panjab (tout comme aussi l'ordre ciśtī), est resté cantonné, comme langue littéraire, à sa région d'origine ${ }^{25}:$ le sikhisme n'en a guère débordé le cadre géographique et c'est dans les diverses langues régionales de l'Inde du Nord qu'ont fleuri des traditions poétiques soufies locales.

Dépendant pour leur composition et/ou pour l'échelle de leur diffusion du patronage respectif de l'empereur, de la secte krishnaïte du puștimārga et des ordres soufis qādirī et ciśtī, les textes d'Abu'l-Fazl, de Surdas et de Bullhe Shah sont aussi en rapport étroit avec des pratiques spécifiques. La chronique impériale écrite en persan par Abu'l-Fazl relève d'un genre codifié de longue date dans les milieux royaux du monde iranisé. Dans ce texte, plus encore que dans beaucoup d'autres de ce genre, le religieux occupe une place cruciale. D'une part, des signes divins annoncent la grandeur de l'empereur et le succès de son règne, notamment dans l'établissement des valeurs fondamentales de l'islam, le discours de divers spécialistes religieux légitime le règne d'Akbar qui les patronne et les honore, et l'empereur a un "programme » dont la double dimension religieuse est l'axe fort : faire régner l'harmonie entre hindous et musulmans, dont les religions respectives sont décrites comme des variantes d'un monothéisme fondamental, et mettre en valeur l'établissement par Akbar d'un nouvel ordre mystique ouvert et conciliant à travers lequel l'empereur affirme son autorité spirituelle. D'autre part, comme il a été dit plus haut à propos de l'introduction de Wheeler M. Thackston, la mystique islamique imprègne de bout en bout le texte de l'Akbarnāma. Les compositions de Surdas et de Bullhe Shah sont pour leur part liées à des pratiques de chant religieux. Dans le cas des poèmes de Surdas, il s'agit du kīrtan, chant de dévotion rituel que pratiquent les fidèles du puṣtimārga dans les « demeures » (havelī) des images de Krishna. Dans celui des kāfī de Bullhe

24. Les hymnes qui constituent le premier livre sacré des sikhs, l'Ādi Granth (compilé en 1604 par leur cinquième Guru, Arjan [1563-1606, Guru à partir de 1581]) sont écrits dans une variété de hindi littéraire mixte, langue religieuse de ceux des bhakta de l'Inde du Nord, collectivement désignés comme sant ( «authentiques »), qui adorent non un Dieu doué d'attributs comme Vishnu ou ses avatāra, mais un Dieu absolu, sans attributs, et dont font précisément partie les sikhs : c'est pourquoi on appelle cette langue sant-bhāṣā, "langue des sants ».

25. La Ciśtiyya fut fondée vers 930 à Chisht, petite ville proche de Hérat dans ce qui est aujourd'hui l'Afghanistan, par Abu Ishaq 'Shami' (le «Syrien », m. Damas, 940), et introduite en Inde par Mu'in al-Din Sijzi (m. Ajmer, Rajasthan, 1230). 
Shah, il s'agit du chant rituel (qavvālī) de poèmes exécuté par des spécialistes (qavvāl) dans les "cours (religieuses) »(dargāh) qui sont les sièges où et d'où s'exerce l'autorité de maîtres soufis régnant sur leurs juridictions spirituelles respectives (vilāya). Regardés ensemble, ces trois corpus donnent une bonne image de la pluralité régionale, sociale, linguistique, culturelle et religieuse qui caractérisait l'Inde moghole tout comme elle demeure un trait marquant de l'Inde d'aujourd'hui.

Les trois volumes dont il a été question dans la présente note sont assurément appelés à devenir des outils indispensables pour nombre d'indianistes, tout comme les deux autres parus en même temps cette année et ceux qui suivront dans la splendide collection de la Murty Classical Library of India. L'accès facile à des classiques soigneusement édités - ou donnés dans la meilleure édition existante - et accompagnés en regard de leur traduction en anglais va graduellement changer le travail de ces chercheurs en les dotant d'outils semblables à ceux dont disposent les occidentalistes. Mais ces trois livres sont aussi, j'espère l'avoir laissé entrevoir, des mines précieuses pour les spécialistes de sciences des religions, tant par la richesse des textes édités et la fiabilité des traductions que par la qualité des introductions et des appareils critiques.

\section{Denis MATRINGE \\ CNRS, Paris \\ Centre d'Études de l'Inde et de l'Asie du Sud (EHESS-CNRS) \\ dmatringe@gmail.com}

\section{Bibliographie}

Abu'L-Fazl, 2015, The History of Akbar, vol. 1, ed. and transl. Thнскsтоn Wheeler M., Cambridge (Ma.), Murty Classical Library of India et Harvard University Press.

AbŪ l-FAŻL-I 'Allāmī, 1873-1887, Akbarnāma, ed. Āghā Aḥmad 'Alī, Bibliothéca Indica 79, 3 vols., Calcutta, Asiatic Society.

Abū l-FaŻl-I 'Allāmī, 1927-1949, Ā'̄̄n-i Akbarī, vol. 1 transl. Blochman Henry, vols. 2 and 3 transl. Jarrett H., $2^{\text {nd }}$ ed. rev. SARKar Jadu Nath, vol. 3 transl. H. S. Jarrett, Calcutta, The Asiatic Society, repr. New Delhi, Oriental Reprint 1977-78.

Anonyme, 2015, Therigatha: Poems of the First Buddhist Women, transl. from Pali by Haluisey Charles, Cambridge (Ma.), Murty Classical Library of India et Harvard University Press.

Bullhe Shah, 2015, Sufi Lyrics, ed. et transl. Shackle Christopher, Cambridge (Ma.), Murty Classical Library of India et Harvard University Press.

CoHN Bernard, 1996, "The Command of Language and the Language of Command ", in CoHn Bernard, Colonialism and Its Forms of Knowledge: The British in India, Princeton, Princeton University Press, p. 16-56.

ENCyClopaedia of Islam III, 2007 (en cours de publication), Leiden, Brill (http:// referenceworks.brillonline.com/browse/encyclopaedia-of-islam-3).

Entwistle Alan W., 1987, Braj, Centre of Krishna Pilgrimage, Groningen, Egbert Forsten. 
Hawley John Stratton, with Goswami Shrivatsa, 1981, At Play with Krishna: Pilgrimage Dramas from Brindavan, Princeton, Princeton University Press, repr. Delhi, Motilal Banarsidass, 1992.

Hawley John Stratton, 1983, Krishna, the Butter Thief, Princeton, Princeton University Press, réimpr. Delhi, Oxford University Press, 1989.

Hawley John Stratton, 1984, Sur Das: Poet, Singer, Saint, Seattle, University of Washington Press, et Delhi, Oxford University Press.

Hawley John Stratton, 2005, Three Bhakti Voices: Mirabai, Surdas, and Kabir in Their Time and Ours, Delhi, Oxford University Press.

Hawley John Stratton, 2009, The Memory of Love: Surdas Sings to Krishna, New York, Oxford University Press.

Hawley John Stratton, 2015, A Storm of Songs: India and the Idea of the Bhakti Movement, Cambridge (MA), Harvard University Press.

Hawley John Stratton, Juergensmeyer Mark, 2004, Songs of the Saints of India, $2^{\text {nd }}$ rev. ed., Delhi, Oxford University Press.

MukherjeE Neel, 2015, "Many Gods, Many Voices: a New Series in Uncovering India's Dazzling Literary History ", New Stateman, 27 février - 5 mars 2015.

Peddana Allasani, 2015, The Story of Manu, transl. from Telugu by Rao Velcheru Narayana and Shulman David, Cambridge (Ma.), Murty Classical Library of India and Harvard University Press.

Pollock Sheldon (ed.), 2003, Literary Cultures in History: Reconstructions from South Asia, Berkeley, University of California Press.

Pollock Sheldon, 2006, The Language of the Gods in the World of Men: Sanskrit, Culture, and Power in Premodern India, Berkeley, University of California Press.

POLLOCK Sheldon, 2015, "Why a Classical Library of India ", http://www.murtylibrary. com/why-a-classical-library-of-india.php.

Richardson E. Allen, 2014, Seing Krishna in America: The Hindu Bhakti Tradition of Vallabhacharya in India and Its Movement to the West, Jefferson (North Carolina), McFarland and Company.

SHACKLE Christopher, 1970, «Punjabi in Lahore », Modern Asian Studies 4/3, p. 239-267.

SHACKLE Christopher, 1972, Punjabi, London, Teach Yourself Books.

SHACKLE Christopher, 1976, The Siraiki Language of Central Pakistan: a Reference Grammar, London, School of Oriental and African Studies.

SHACKLE Christopher, 1977a, "Siraiki: A Language Movement in Pakistan ", Modern Asian Studies 11/3, p. 379-403.

SHACKLE Christopher, 1977b, "South-Western" Elements in the Language of the $\bar{A} d i$ Granth", Bulletin of the School of Oriental and African Studies 40/1, p. 36-50.

SHACKLE Christopher, 1978a, "The South Western Style in the Guru Granth Sabib ", Journal of Sikh Studies 5/1, p. 69-87.

SHACKLE Christopher, 1978b, "Approaches to the Persian Loans in the Ädi Granth ", Bulletin of the School of Oriental and African Studies 41/1, p. 73-96.

SHACKLE Christopher, 1978c, "The Sahaskritī Poetic Idiom in the Ādi Granth ", Bulletin of the School of Oriental and African Studies 4/2, p. 297-313.

SHACKLE Christopher, 1978d, "The Multani “Marsiya” ", Islam 55 (1978), p. 281-311.

SHACKLE Christopher (trad.), 1983, Fifty Poems of Khawaja Farid, Multan, Bazm-eSaqafat. 
SHACKLE Christopher, 1984, An Introduction to the Sacred Language of the Sikhs, Londres, School of Oriental and African Studies.

SHACKLE Christopher, 1992, "Transitions and Transformations in Vāris Shāh's Hìr ", The Indian Narrative: Perspectives and Patterns, ed. with SHACKLE Christopher and SNELl Ruppert, Wiesbaden, Harrassowitz, p. 241-263.

SHACKLE Christopher, 1985, Hashim Shah: Sassi, Lahore, Vanguard Books.

SHACKLE Christopher, 1993, "Early Vernacular Poetry in the Indus Valley: Its Contexts and Character ", in Piccola Anna Della, Zingel-Avé-Lallemant Stephanie (eds.), Islam and Indian Regions, Beiträge zur Südasien-Forschung, Südasien-Institut Universität Heidelberg 145, 2 vols., Wiesbaden, Franz Steiner Verlag, 1993, I, p. 417-443.

SHACKLE Christopher, 1995a, A Gurū Nānak Glossary, $2^{\text {nd }}$ ed. (including The NonSanskritic Vocabulary of the Later Sikh Gurus), New Delhi, Heritage Publishers.

SHACKLE Christopher, 2000, "Beyond Turk and Hindu: Crossing the Boundaries in IndoMuslim Romance ", in Gilmartin David, Lawrence Bruce B. (eds.), Beyond Turk and Hindu: Rethinking Identities in Islamicate South Asia, Gainesville, University Press of Florida, p. 55-73.

SHACKLE Christopher (ed. and transl., with MandaIR A. S.), 2005, Teachings of the Sikh Gurus: Selections from the Sikh Scriptures, London and New York, Routledge.

SHaCkLE Christopher, 2006, "The Shifting Sands of Love ", in Orsini Francesca (ed.), Love in South Asia: A Cultural History, Cambridge, Cambridge University Press, p. 87-108.

SHACKLE Christopher, 2007, "The Story of Sayf al-Muluk in South Asia ", Journal of the Royal Asiatic Society of Great Britain \& Ireland 17/2, p. 115-129.

ShaCKLE Christopher, 2011, "Panjabi Sufi Poetry from Farid to Farid », in Malhotra Ansu, Mir Farina (eds.), Punjab Reconsidered: History, Culture and Practice, New Delhi, Oxford University Press, p. 3-34.

SOUR-DAS, 1971, Pastorales, traductions de la langue braj avec introduction, notes et glossaire par VAudeville Charlotte, Paris, Gallimard-UNESCO, "Connaissance de l'Orient ", $\mathrm{n}^{\circ} 35$.

STREUSAND Douglas, 2007-... (a), "Akbar », in Encyclopaedia of Islam III.

STReuSAND Douglas, 2007-... (b), « Abū l-Faḍl-i 'Allāmī », in Encyclopaedia of Islam III.

SuRdAs, 2015, Sur's Ocean: Poems from the Early Tradition, ed. BRYANT Kenneth E., transl. Hawley John Stratton, Cambridge (Ma.), Murty Classical Library of India et Harvard University Press.

ThAскSTON Wheeler M. (transl.), 1999, The Jahangirnama: Memoirs of Jhangir, Emperor of India, New York, Oxford University Press.

Thackston Wheeler M. (transl.), 2002, The Baburnama: Memoirs of Babur, New York, Modern Library Classics.

Thackston Wheeler M. (transl.), 2009, Three Memoirs of Humayun, Costa Mesa (CA), Mazda Publishers.

VAudeVILle Charlotte, 1976, "Braj, lost and found ", Indo-Iranian Journal 18/3, p. 195213. 